\title{
Heat-Induced, Pressure-Induced and Centrifugal-Force-Induced Exact Axisymmetric Thermo-Mechanical Analyses in a Thick-Walled Spherical Vessel, an Infinite Cylindrical Vessel, and a Uniform Disc Made of an Isotropic and Homogeneous Material
}

\author{
Vebil Yıldırım \\ University of Çukurova, Department of Mechanical Engineering, 01330, Adana, TURKEY \\ E-mail address: vebil@cu.edu.tr \\ Received date: April 2017 \\ Accepted Date: May 2017
}

\begin{abstract}
Heat-induced, pressure-induced, and centrifugal force-induced axisymmetric exact deformation and stresses in a thick-walled spherical vessel, a cylindrical vessel, and a uniform disc are all determined analytically at a specified constant surface temperature and at a constant angular velocity. The inner and outer pressures are both included in the formulation of annular structures made of an isotropic and homogeneous linear elastic material. Governing equations in the form of Euler-Cauchy differential equation with constant coefficients are solved and results are presented in compact forms. For discs, three different boundary conditions are taken into account to consider mechanical engineering applications. The present study is also peppered with numerical results in graphical forms.
\end{abstract}

Keywords: Thermo-Mechanical, Elasticity solution, Exact solution, Rotating disc, Pressure vessel, Linear elastic

\section{Introduction}

Annular structures such as cylindrical or spherical vessels including discs are essential structural elements mainly made of an isotropic and homogeneous material. (Fig. 1). From those vessels may store gases, vapors, and liquids at various pressures and temperatures. The pressure is obtained from an external source, or by the application of heat from an indirect or direct source. That is a pressure vessel is mostly subjected simultaneously to both the mechanical and thermal loads. In a pressure vessel design determination of both the displacements and stresses is of great importance. If the material of the vessel is isotropic and homogeneous then those may be calculated analytically. By choosing appropriate parameters, an analytical solution also allows the optimization of the design parameters of a vessel structure.

Apart from vessels, a rotating disc is also one of the essential annular structural component. They are commonly used in a wide variety of engineering applications including space structures, electronic components and rotating machinery. Axisymmetric elasticity solutions to the both mechanical and thermal stress analysis of rotating discs have long been studied in the available literature. However, most of those studies modelled the thermo-elastic behavior of a disc with boundary condition which commonly proper 
for the cylindrical vessel having stress-free surfaces (Fig. 1c). But, in mechanical engineering applications rotating discs are commonly attached a rigid shaft at the center (Figs. 1d-e).

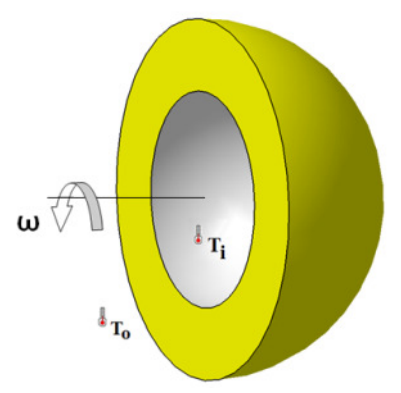

(a) Sphere
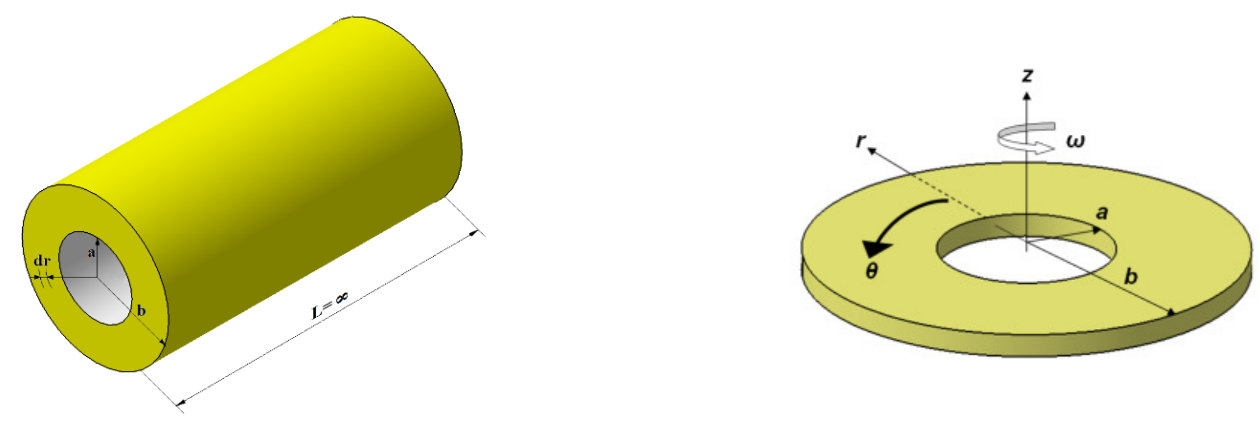

b) Infinite cylinder

c) Disc / Circular annulus
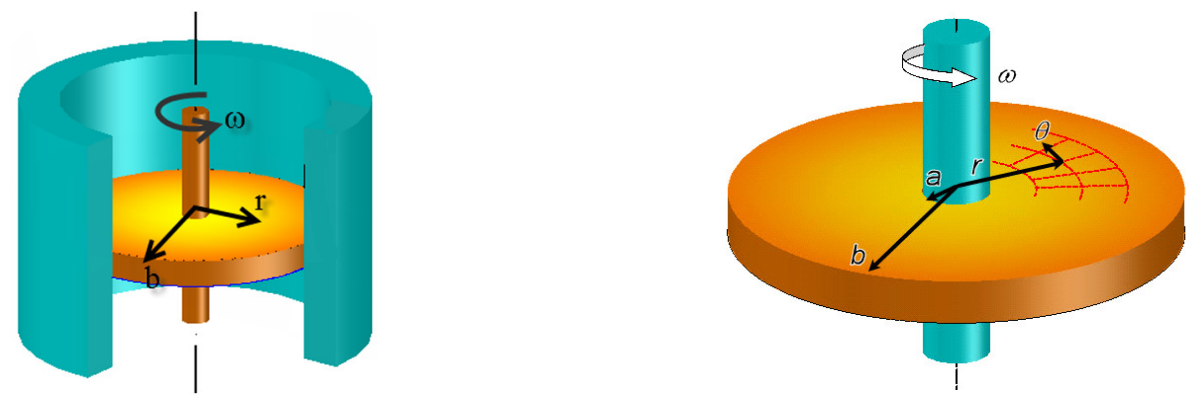

d) Disc having rigid case at the outer surface

e) Disc mounted a shaft at its center

Fig. 1. Rotating annular structural geometries 
As is well known in the thin-walled structure analysis the uniform stress distribution along the thickness is taken for granted. Apart from this, the effect of the radial stress on the equivalent stress is neglected. That is the radial stress due to either/both inner or/and outer pressures are assumed to be virtually zero.

However, in thick-walled structures, both the radial and hoop stresses play a role in the vessel design. It is obvious that the distribution of the stresses along the radial coordinate are no further uniform in thickwalled annular structures.

In the literature, the most number of studies are conducted with such structures subjected to just internal pressure. However, there are some types of structures such as submarine structures and vacuum tanks for which the predominant pressure is assumed to be the outer pressure and just the effect of this external pressure is considered in their analysis. In the present study effects of both the inner and the outer pressures are formulated analytically for each type of annular structures.

In some thermal studies, for the aim of simplicity, the distribution of the temperature along the radial coordinate is assumed to be linear without solving related Fourier heat conduction differential equation in thick-walled annular structures. As might be expected, this not reflects the true thermal behavior of such structures. The appropriate temperature distribution, which is obtained in terms of a logarithmic function, is identically the same but not linear for discs and cylindrical structures (Fig. 2). The temperature distribution in spheres shows a hyperbolic variation. In the present study, the exact temperature distributions obtained by the solution of Fourier heat conduction differential equation are used to study the thermo-elastic behaviors of such structures.

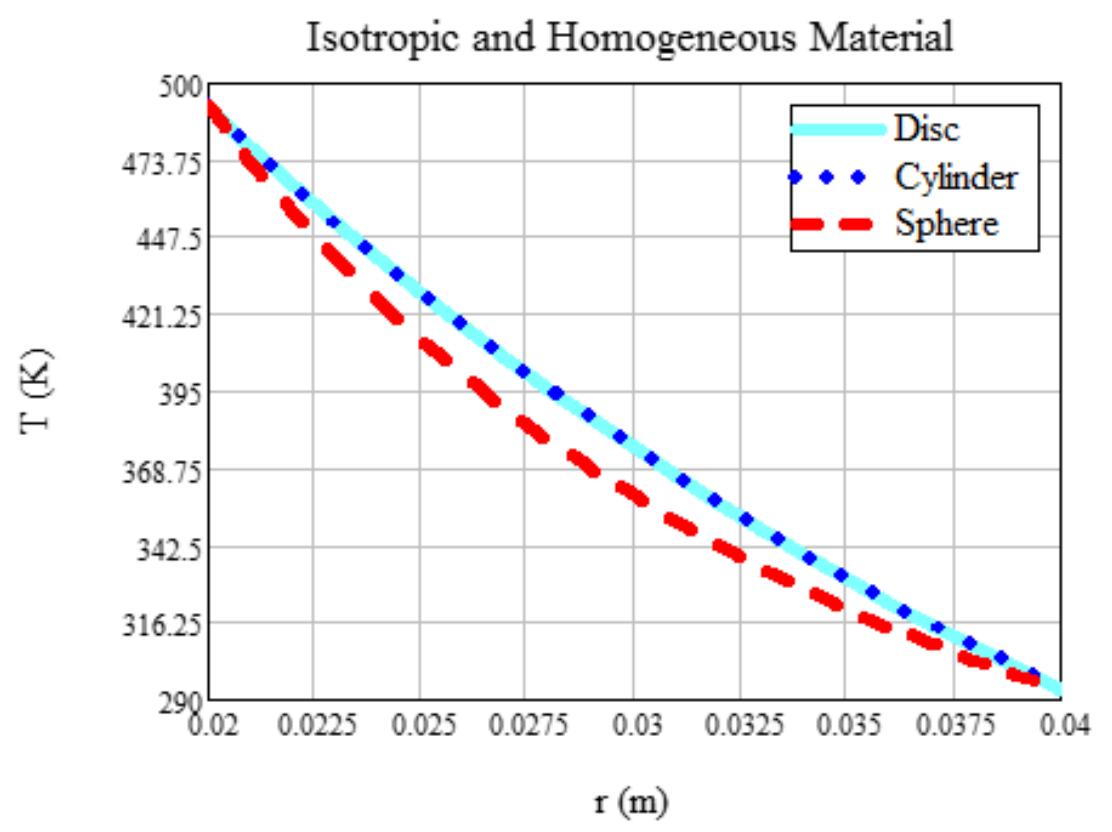

Fig. 2. Temperature distribution in thick-walled annular structures 
Apart from the above, one may also be confused undoubtedly when studying the disc and cylindrical geometries. Discs are modeled in the case of plane stress assumption while the cylinders are modeled under plane-strain assumptions. The strain-displacement relations together with the equilibrium equation are identically the same under axisymmetric conditions for two annular structural types. As stated above, the temperature distribution of two types of structures are also one and the same. In spite of those, there are differences in their stress-strain relations that is in Hooke's law. This, sometimes, may cause some misperceptions in the formulation. In the present study the main differences in the formulation are demonstrated clearly.

Finally, one may spend relatively much time to obtain formulas with the same notation for thermomechanical behavior of such structures. In this respect, this study offers a concise and a complete study.

The subject of the present work is to form an infallible all-in-one source for the linear elastic behavior of such structures made of an isotropic and homogeneous material under thermal and mechanical loads (Fig. 1). Centrifugal forces, internal and external pressure forces are all classified as mechanical loads. To do so, governing equations which are second degree order non-homogeneous differential equations of constant coefficients are first derived from the elasticity field equations, and then they are solved analytically to obtain thermal and mechanical deformation and stresses. In this study exact thermomechanical analysis of this types of structures are carried out according to the superposition principle since small displacements are assumed. That is, each elastic quantity, either displacement or stress, is first determined separately for the related loading type. The resultant elastic quantity is then determined as a sum of each contributions.

$$
\begin{aligned}
& \left(\sigma_{\theta}\right)_{\text {THERMO-MECHANICAL }}=\left(\sigma_{\theta}\right)_{\text {Pressure }}+\left(\sigma_{\theta}\right)_{\text {Rotation }}+\left(\sigma_{\theta}\right)_{\text {Thermal }} \\
& \left(u_{r}\right)_{\text {THERMO-MECHANICAL }}=\left(u_{r}\right)_{\text {Pressure }}+\left(u_{r}\right)_{\text {Rotation }}+\left(u_{r}\right)_{\text {Thermal }} \\
& \left(\sigma_{r}\right)_{\text {THERMO-MECHANICAL }}=\left(\sigma_{r}\right)_{\text {Pressure }}+\left(\sigma_{r}\right)_{\text {Rotation }}+\left(\sigma_{r}\right)_{\text {Thermal }}
\end{aligned}
$$

\section{Spherical Vessels}

In a spherical coordinate system, $(r, \theta, \emptyset)$, relations between the strain and displacement components for spherically symmetric case are as follows (see Notations)

$$
\begin{gathered}
\varepsilon_{r}(r)=u_{r}{ }^{\prime}(r) \\
\varepsilon_{\theta}(r)=\varepsilon_{\phi}(r)=\frac{u_{r}(r)}{r}
\end{gathered}
$$




$$
\gamma_{r \theta}(r)=\gamma_{r \phi}(r)=\gamma_{\theta \phi}(r)=0
$$

where prime symbol denotes the first derivative of the quantity with respect to the radial coordinate. It may be noted that the properties in $\theta$ and $\varnothing$ directions are identical for axisymmetric hollow spheres. Denoting the rise in temperature with respect to the temperature where stress value in the material is zero by $\Delta T(r)=T-T_{\infty}$, Hooke's law for a sphere made of an isotropic and homogeneous material is given by

$$
\begin{gathered}
\sigma_{r}(r)=C_{11} \varepsilon_{r}(r)+C_{12} \varepsilon_{\theta}(r)+C_{12} \varepsilon_{\phi}(r)-\left(C_{11}+2 C_{12}\right) \alpha \Delta T(r) \\
=C_{11} \varepsilon_{r}(r)+2 C_{12} \varepsilon_{\theta}(r)-\frac{E}{1-2 v} \alpha \Delta T(r) \\
=C_{11} \varepsilon_{r}(r)+2 \lambda C_{11} \varepsilon_{\theta}(r)-(1+2 \lambda) C_{11} \alpha \Delta T(r) \\
\begin{array}{r}
\sigma_{\theta}(r)=\sigma_{\phi}(r)=C_{12} \varepsilon_{r}(r)+\left(C_{11}+C_{12}\right) \varepsilon_{\theta}(r)-\left(C_{11}+2 C_{12}\right) \alpha \Delta T(r) \\
=C_{12} \varepsilon_{r}(r)+\left(C_{11}+C_{12}\right) \varepsilon_{\theta}(r)-\frac{E}{1-2 \nu} \alpha \Delta T(r) \\
=\lambda C_{11} \varepsilon_{r}(r)+(1+\lambda) C_{11} \varepsilon_{\theta}(r)-(1+2 \lambda) C_{11} \alpha \Delta T(r)
\end{array}
\end{gathered}
$$

Where

$$
C_{11}=\frac{(1-v) E}{(1-2 v)(1+v)} ; \quad C_{12}=\frac{v E}{(1-2 v)(1+v)}=\frac{v}{1-v} C_{11}=\lambda C_{11}
$$

Equilibrium equation for a spherical vessel rotating at a constant angular velocity is

$$
\sigma_{r}^{\prime}(r)+\frac{2}{r}\left(\sigma_{r}-\sigma_{\theta}\right)=-\rho \omega^{2} r
$$

Eqs. (2), (3), and (5) are referred to as the field equations of the elasticity. Substituting Eq. (2) into Eq. (3), and then successive substitution of Eq. (3) together with the first derivative of the radial stress into the equilibrium equation (5), the governing equation called Navier equation in terms of radial displacement is obtained as follow

$$
u_{r}^{\prime \prime}(r)+\frac{2}{r} u_{r}^{\prime}(r)-\frac{2}{r^{2}} u_{r}(r)=-\frac{\rho \omega^{2} r}{C_{11}}+(1+2 \lambda) \alpha T^{\prime}(r)=-\frac{\rho \omega^{2} r}{C_{11}}+\frac{(1+v)}{(1-v)} \alpha T^{\prime}(r)
$$

This is a second order non-homogeneous Euler-Cauchy type differential equation with constant coefficient. Its solution consists of the sum of its homogeneous and particular solutions. Since small displacements are assumed, the superposition principle holds.

To consider just mechanical loads due to either internal or external pressures, the following $(\omega=\Delta T=$ $0)$ is solved with the boundary conditions [1]: $\sigma_{r}(a)=-p_{a}$, and $\sigma_{r}(b)=-p_{b}$. 


$$
u_{r}^{\prime \prime}(r)+\frac{2}{r} u_{r}^{\prime}(r)-\frac{2}{r^{2}} u_{r}(r)=0
$$

In order to account for just the rotation as a mechanical load ( $\left.p_{a}=p_{b}=0 ; \Delta T=0\right)$, Eq. (8) is solved under the boundary conditions: $\sigma_{r}(a)=0$ and $\sigma_{r}(b)=0$.

$$
u_{r}^{\prime \prime}(\mathrm{r})+\frac{2}{r} u_{r}^{\prime}(r)-\frac{2}{r^{2}} u_{r}(r)=-\frac{\rho \omega^{2} r}{C_{11}}
$$

After determination of the temperature distribution along the thickness of the sphere, the thermo-elastic analysis is merely taken into consideration by the following [2-6] under the boundary conditions: $\sigma_{r}(a)=$ $0 ; \sigma_{r}(b)=0$.

$$
u_{r}^{\prime \prime}(r)+\frac{2}{r} u_{r}^{\prime}(r)-\frac{2}{r^{2}} u_{r}(r)=\frac{(1+v)}{(1-v)} \alpha T^{\prime}(r)
$$

As stated above, before conducting the thermo-elastic analysis, a thermal analysis which defines the distribution of the temperature along the radial coordinate is required. Under the steady-state condition, in the absence of heat generation, temperature distribution along the thickness of the spherical vessel is found from the solution of the following heat conduction equation (Fourier's equation) with the first kind boundary conditions (Dirichlet): $T(a)=T_{a}$ and $T(b)=T_{b}$.

$$
T^{\prime \prime}(r)+\frac{2}{r} T^{\prime}(r)=0
$$

Solution of the above is found as

$$
\begin{gathered}
T(r)=-\frac{D_{1}}{r}+D_{2} \\
D_{1}=\frac{a b\left(T_{a}-T_{b}\right)}{a-b}=\frac{b\left(T_{a}-T_{b}\right)}{1-\frac{b}{a}} ; \quad D_{2}=\frac{a T_{a}-b T_{b}}{a-b}=\frac{T_{a}-\frac{b}{a} T_{b}}{1-\frac{b}{a}} \\
T(r)=\frac{-b r T_{b}+a\left(r T_{a}+b\left(T_{b}-T_{a}\right)\right)}{(a-b) r}=\frac{a(r-b) T_{a}+b(a-r) T_{b}}{(a-b) r}=-\frac{a b\left(T_{a}-T_{b}\right)}{(a-b) r}+\frac{a T_{a}-b T_{b}}{a-b}
\end{gathered}
$$

Eq. (9), now, takes the following form with Eq. (11)

$$
u_{r}^{\prime \prime}(r)+\frac{2}{r} u_{r}^{\prime}(r)-\frac{2}{r^{2}} u_{r}(r)=\frac{a b\left(T_{a}-T_{b}\right) \alpha(1+v)}{(a-b) r^{2}(1-v)}=\frac{\Psi}{r^{2}}
$$

Solution of the above inhomogeneous equation with the boundary conditions, $\sigma_{r}(a)=0$, and $\sigma_{r}(b)=0$, gives the following

$$
\begin{gathered}
u_{r}(r)=\frac{B_{2}}{r^{2}}+B_{1} r-\frac{\Psi}{2} \\
B_{1}=\frac{\lambda(v-1) \Psi(a-b)(a+b)-\alpha(v+1)\left(a^{3} T_{a}-b^{3} T_{b}\right)}{(2 \lambda+1)(v-1)\left(a^{3}-b^{3}\right)}
\end{gathered}
$$


V. Yıldirım

$$
B_{2}=\frac{a^{2} b^{2}\left(\lambda(v-1) \Psi(a-b)+a b \alpha(v+1)\left(T_{a}-T_{b}\right)\right)}{2(\lambda-1)(v-1)\left(a^{3}-b^{3}\right)}
$$

Compact forms of the thermo-elastic radial displacement, radial and hoop stresses are

$$
\begin{gathered}
u_{r}(r)=\frac{K}{2(v-1) r^{2}\left(a^{3}-b^{3}\right)} \\
K=\alpha\left(a^{3}\left(b^{3}(v+1)\left(-\left(T_{a}-T_{b}\right)\right)+b(v+1) r^{2}\left(T_{a}-T_{b}\right)+2(v-1) r^{3} T_{a}\right)\right. \\
+a^{2} b r^{2}\left(T_{a}-T_{b}\right)(b v+b-2 v r)+a b^{2} r^{2}\left(T_{a}-T_{b}\right)(b v+b-2 v r) \\
\left.-2 b^{3}(v-1) r^{3} T_{b}\right) \\
\sigma_{r}(r)=\frac{a b \alpha E(a-r)(b-r)\left(T_{a}-T_{b}\right)(a(b+r)+b r)}{(v-1) r^{3}\left(a^{3}-b^{3}\right)} \\
\sigma_{\theta}(r)=-\frac{a b \alpha E\left(T_{a}-T_{b}\right)\left(r^{2}\left(a^{2}+a b+b^{2}\right)+a^{2} b^{2}-2 r^{3}(a+b)\right)}{2(v-1) r^{3}\left(a^{3}-b^{3}\right)}
\end{gathered}
$$

Nayak et al. [4] offered the following thermal stresses for hollow spheres.

$$
\begin{gathered}
\sigma_{r}(r)=\frac{-\alpha E\left(T_{a}-T_{b}\right)}{(1-v)}\left\{\frac{\frac{b}{r}-1}{\frac{b}{a}-1}-\frac{\frac{b^{3}}{r^{3}}-1}{\frac{b^{3}}{a^{3}}-1}\right\}=\sigma_{r-\operatorname{Present}(E q .14)} \\
\sigma_{\theta}(r)=\frac{-\alpha E\left(T_{a}-T_{b}\right)}{(1-v)}\left\{\frac{\frac{b}{2 r}-1}{\frac{b}{a}-1}+\frac{\frac{b^{3}}{2 r^{3}}+1}{\frac{b^{3}}{a^{3}}-1}\right\}=\sigma_{\theta-\text { Present }} \text { Eq.14) }
\end{gathered}
$$

Nayak et al. [4] stated that from References [5-6] one can easily verify that Eq. (15) is indeed the expression for radial and tangential stresses for an isotropic and homogeneous thick spherical vessel. It is also readily verified that Nayak et al.'s [4] equations in (15) and present equations in (14) are identical. For the mechanical load due to internal and external pressures, analytical solution is found as

$$
\begin{gathered}
u_{r}(r)=\frac{C_{2}}{r^{2}}+C_{1} r \\
\sigma_{r}=\frac{C_{11}\left(2 C_{2}(-1+\lambda)+C_{1} r^{3}(1+2 \lambda)\right)}{r^{3}} \\
\sigma_{\theta}=\frac{C_{11}\left(C_{2}-C_{2} \lambda+C_{1} r^{3}(1+2 \lambda)\right)}{r^{3}} \\
C_{1}=-\frac{\left(2 v^{2}+v-1\right)\left(a^{3} p_{a}-b^{3} p_{b}\right)}{E(2 \lambda+1)(v-1)\left(a^{3}-b^{3}\right)} ; C_{2}=\frac{a^{3} b^{3}\left(2 v^{2}+v-1\right)\left(p_{a}-p_{b}\right)}{2 E(\lambda-1)(v-1)\left(a^{3}-b^{3}\right)}
\end{gathered}
$$


Compact forms of the above in which radial and hoop stresses coincide with Roark's formulas [2] are.

$$
\begin{gathered}
u_{r}(r)=-\frac{a^{3} p_{a}\left(b^{3}(v+1)+2(1-2 v) r^{3}\right)}{2 r^{2}\left(a^{3}-b^{3}\right) E}+\frac{b^{3} p_{b}\left(a^{3}(v+1)+2(1-2 v) r^{3}\right)}{2 r^{2}\left(a^{3}-b^{3}\right) E} \\
\sigma_{r}(r)=\frac{a^{3} p_{a}\left(b^{3}-r^{3}\right)}{\left(a^{3}-b^{3}\right) r^{3}}+\frac{b^{3} p_{b}\left(r^{3}-a^{3}\right)}{\left(a^{3}-b^{3}\right) r^{3}} \\
\sigma_{\theta}(r)=-\frac{a^{3} p_{a}\left(b^{3}+2 r^{3}\right)}{2\left(a^{3}-b^{3}\right) r^{3}}+\frac{b^{3} p_{b}\left(a^{3}+2 r^{3}\right)}{2\left(a^{3}-b^{3}\right) r^{3}}
\end{gathered}
$$
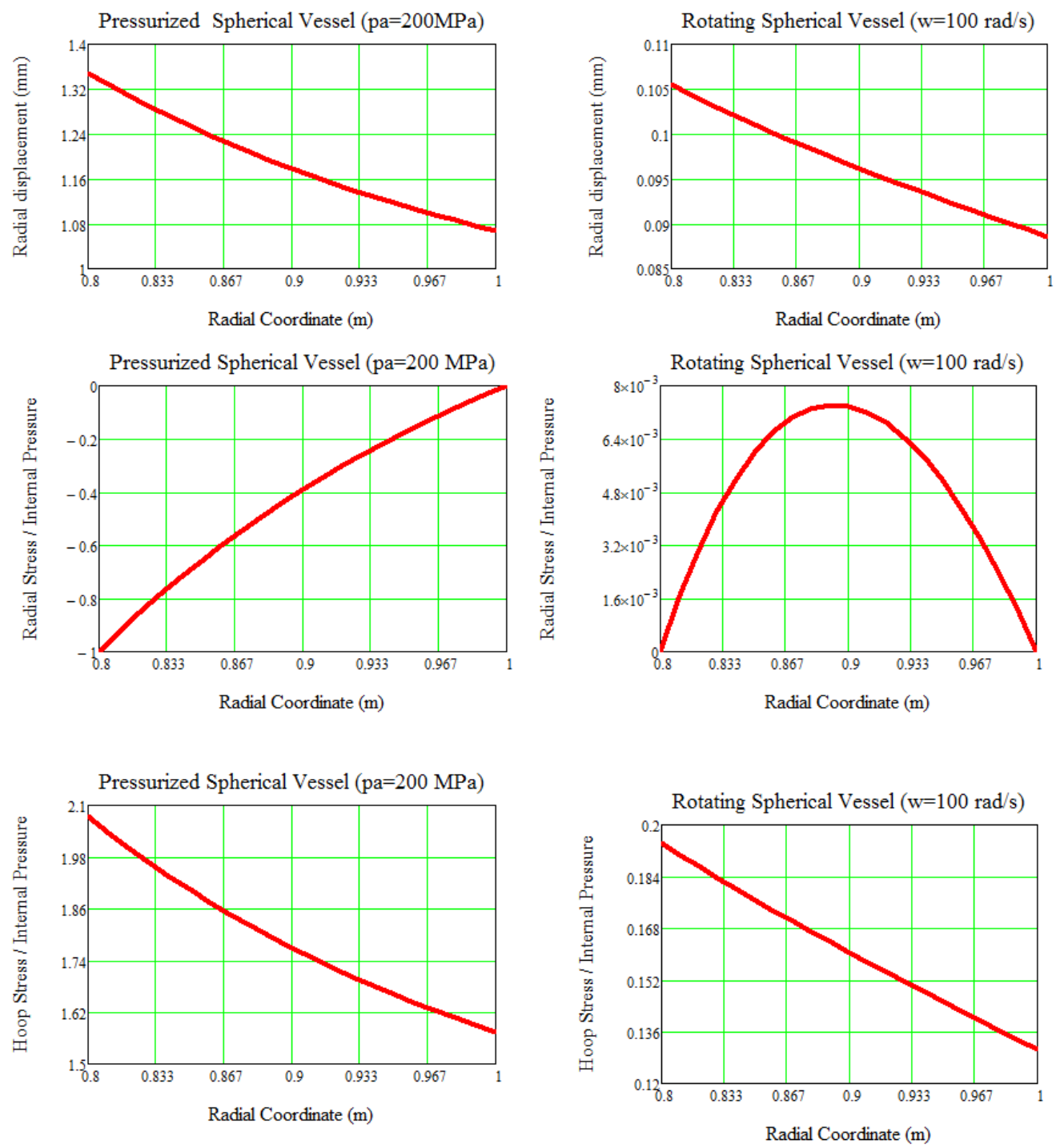

Fig. 3. Displacements and stresses induced by mechanical loads 

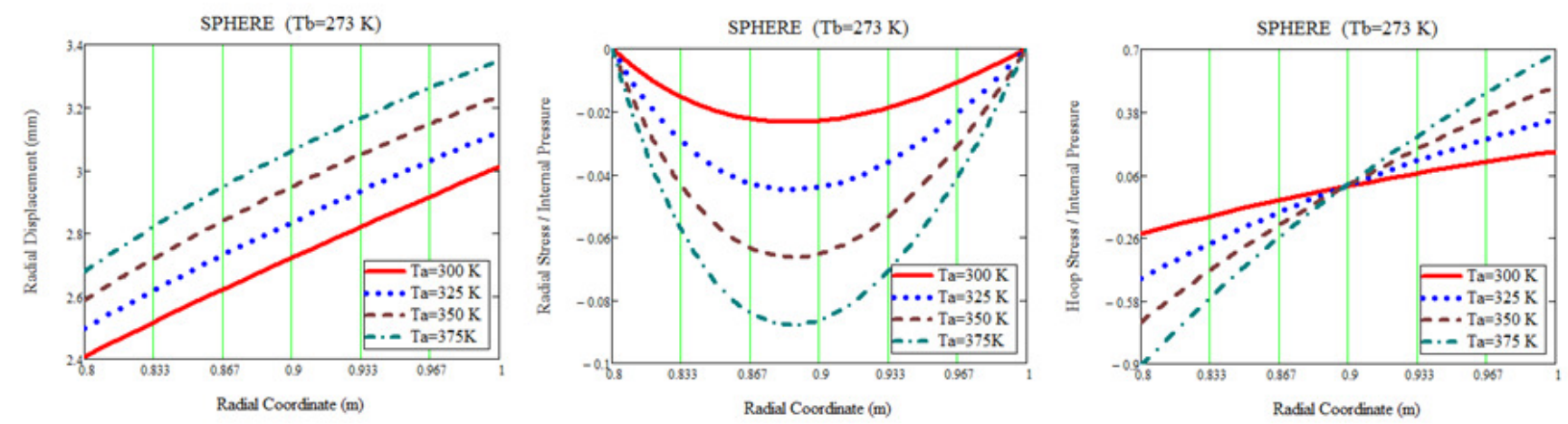

Fig. 4. Displacements and stresses induced by thermal loads

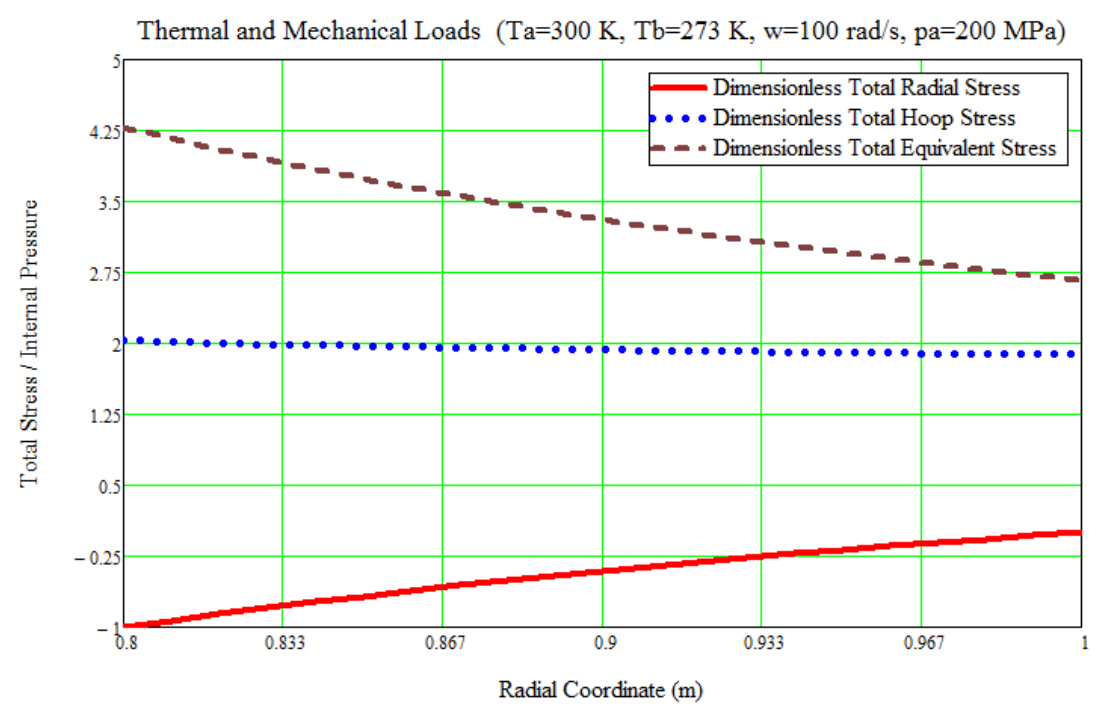

(a)

Fig. 5. Total and equivalent stresses for thermo-mechanical loads

Analytical solutions for mechanical load due to just rotation at a constant angular velocity is

$$
\begin{gathered}
u_{r}(r)=\frac{A_{2}}{r^{2}}+A_{1} r-r^{3} \Omega \\
\sigma_{r}(r)=\frac{2 C_{11} A_{2}(-1+\lambda)}{r^{3}}+A_{1} C_{11}(1+2 \lambda)-C_{11} r^{2}(3+2 \lambda) \Omega \\
\sigma_{\theta}(r)=-\frac{C_{11} A_{2}(-1+\lambda)}{r^{3}}+A_{1} C_{11}(1+2 \lambda)-C_{11} r^{2}(1+4 \lambda) \Omega \\
\Omega=\frac{\left(2 v^{2}+v-1\right) \rho \omega^{2}}{10 E(v-1)} \\
A_{1}=\frac{\left(a^{4}+b a^{3}+b^{2} a^{2}+b^{3} a+b^{4}\right)(2 \lambda+3) \Omega}{\left(a^{2}+b a+b^{2}\right)(2 \lambda+1)} \quad A_{2}=-\frac{a^{3} b^{3}(a+b)(2 \lambda+3) \Omega}{2\left(a^{2}+b a+b^{2}\right)(\lambda-1)}
\end{gathered}
$$


For a numerical example, geometrical and material properties together with boundary conditions of the sphere are assumed to be [4]:

$$
\begin{gathered}
E=209.2 \mathrm{GPa} ; \quad v=0.29 ; \quad \sigma_{\text {yield }}=700 \mathrm{MPa} ; \alpha=10.5810^{-6} 1 /^{\circ} \mathrm{C} \\
T_{a}=27^{\circ} \mathrm{C} ; T_{b}=0^{\circ} \mathrm{C} ; p_{a}=200 \mathrm{MPa} ; p_{b}=0 ; \omega=100 \mathrm{rad} / \mathrm{s} ; a=0.8 \mathrm{~m} ; b=1.0 \mathrm{~m}
\end{gathered}
$$

Variation of the displacements and stresses induced by separate mechanical and thermal loads are illustrated in Figs. 3-4. From these figures it is observed that the radial displacement and hoop stresses which are tension in nature decrease with increasing $b / a$ ratios for each individual mechanical loads. The maximum radial stress which is compression in nature is observed at the inner surface for mechanical pressure loads, and at the vicinity of the middle surface as being tension in nature for mechanical rotational loads. Variation of the displacements and stresses induced by thermal loads is illustrated in Fig. 4 at different temperatures of the inner surface. From the figure it is observed that the radial displacement increases with increasing $b / a$ ratios and with increasing inner surface temperature. The maximum radial stress in compression is observed at the vicinity of the middle surface and increases with increasing surface temperature differences. Tangential stress varies from compressive to tensile for thermal load, from inside surface to outside. Considering superposition principle, variation of the thermo-mechanical stresses and equivalent stress in Eq. (19) which is given by [4] based on the Von-Mises criteria is illustrated in Fig. 5. It is observed that the equivalent stress gradually decreases in the radial direction, from inside surface to outside for thermo- mechanical loads and sets up tensile stresses. From this figure it is also observed that the equivalent stress exceeds the yield strength at the inner surface, $\sigma_{\text {yield }} / p_{a}=3.5$.

$$
\sigma_{e q}=\sqrt{2}\left(\sigma_{\theta}-\sigma_{r}\right)
$$

\section{Cylindrical Vessels}

In a polar coordinate system, $(r, \theta)$, axisymmetric relations between the strain and displacement components are as follows (Fig. 1)

$$
\varepsilon_{r}(r)=u_{r}{ }^{\prime}(r) ; \quad \varepsilon_{\theta}(r)=\frac{u_{r}(r)}{r} ; \quad \gamma_{r \theta}(r)=0
$$

Stress-strain relations for a cylindrical structure are given in the form of

$$
\begin{gathered}
\sigma_{r}(r)=C_{11} \varepsilon_{r}(r)+C_{12} \varepsilon_{\theta}(r)-\left(C_{11}+2 C_{12}\right) \alpha \Delta T(r)=C_{11} \varepsilon_{r}(r)+\lambda C_{11} \varepsilon_{\theta}(r)-(1+2 \lambda) C_{11} \alpha \Delta T(r) \\
\sigma_{\theta}(r)=C_{12} \varepsilon_{r}(r)+C_{11} \varepsilon_{\theta}(r)-\left(C_{11}+2 C_{12}\right) \alpha \Delta T(r)=\lambda C_{11} \varepsilon_{r}(r)+C_{11} \varepsilon_{\theta}(r)-(1+2 \lambda) C_{11} \alpha \Delta T(r) \\
C_{11}=\frac{(1-v) E}{(1-2 v)(1+v)} ; \quad C_{12}=\frac{v E}{(1-2 v)(1+v)}=\frac{v}{1-v} C_{11}=\lambda C_{11}
\end{gathered}
$$


Equilibrium equation for a cylindrical vessel or a disc rotating at a constant angular velocity, is

$$
\sigma_{r}{ }^{\prime}(r)+\frac{1}{r}\left(\sigma_{r}-\sigma_{\theta}\right)=-\rho \omega^{2} r
$$

Substituting Eqs. (20) into Eqs. (21), and then successive substitution of Eqs. (21) with the first derivative of radial stress into the equilibrium equation in (22), a second order non-homogeneous Navier differential equation which governs the thermo-mechanical behavior of a cylindrical vessel is obtained as follows

$$
u_{r}^{\prime \prime}(r)+\frac{1}{r} u_{r}^{\prime}(r)-\frac{1}{r^{2}} u_{r}(r)=-\frac{\rho \omega^{2} r}{C_{11}}+(1+2 \lambda) \alpha T^{\prime}(r)
$$

In order to study thermo-elastic analysis alone of such structures, let's neglect the rotation together with inner/outer pressures

$$
u_{r}^{\prime \prime}(r)+\frac{1}{r} u_{r}^{\prime}(r)-\frac{1}{r^{2}} u_{r}(r)=(1+2 \lambda) \alpha T^{\prime}(r)
$$

Solution of the above equation consists of the sum of its homogeneous and particular solutions. To get the particular solution, first, the temperature distribution due to the temperature difference between the cylinder surfaces at specific temperatures is required. Let's consider the Fourier heat conduction equation in polar coordinates for cylinders or discs

$$
\frac{1}{r} \frac{d}{d r}\left(\frac{d T(r)}{d r}\right)=T^{\prime \prime}(r)+\frac{1}{r} T^{\prime}(r)=0
$$

Temperature distribution along the thickness of a cylinder or a disc is found from the solution of the above equation with the first kind boundary conditions: $T(a)=T_{a}$ and $T(b)=T_{b}$.

$$
\begin{aligned}
& T_{\text {Cylinder }}(r)=T_{\text {Disk }}(r)=\ln r \Theta_{1}+\Theta_{2} \\
& \Theta_{1}=\frac{T_{a}-T_{b}}{\ln a-\ln b} \quad ; \Theta_{2}=\frac{-\ln b T_{a}+\ln a T_{b}}{\ln a-\ln b}
\end{aligned}
$$

It may be noted that the temperature distribution in both cylinder and disc is govern by the same differential equation under the same boundary conditions. Considering the temperature distribution in Eq. (26) and its derivative, Navier equation for the thermo-elastic analysis of a cylindrical vessel made of a homogeneous and isotropic material is achieved as follows

$$
u_{r}^{\prime \prime}(r)+\frac{1}{r} u_{r}^{\prime}(r)-\frac{1}{r^{2}} u_{r}(r)=(1+2 \lambda) \alpha T^{\prime}(r)=(1+2 \lambda) \frac{\alpha}{r}\left(\frac{T_{a}-T_{b}}{\ln a-\ln b}\right)
$$

In the present work, the above differential equation is solved for the boundary conditions: $\sigma_{r}(a)=0$ and $\sigma_{r}(b)=0$. Solution of Eq. (27) is obtained as follows 
V. Ylldırım

$$
\begin{aligned}
& u_{r}(r)=-\frac{H}{2(v-1) r(a-b)(a+b)(\log (a)-\log (b))} \\
H= & \left\{( v + 1 ) \alpha \left(T _ { a } \left(a^{2} \log (a)\left(b^{2}-2 v r^{2}+r^{2}\right)-b^{2} \log (b)\left(a^{2}-2 v r^{2}+r^{2}\right)\right.\right.\right. \\
+ & +(v-1) r^{2}(a-b)(a+b)+2(v-1) r^{2}(a-b)(a+b) \log (b) \\
& \left.+r^{2}(a-b)(a+b) \log (r)\right) \\
& +T_{b}\left(a^{2} \log (a)\left(-\left(b^{2}-2 v r^{2}+r^{2}\right)\right)+b^{2} \log (b)\left(a^{2}-2 v r^{2}+r^{2}\right)\right. \\
& +r^{2}\left(b^{2}-a^{2}\right) \log (r)-(v-1) r^{2}(a-b)(a+b) \\
& \left.\left.\left.-2(v-1) r^{2}(a-b)(a+b) \log (a)\right)\right)\right\} \\
\sigma_{r}(r)= & \frac{\left(T_{a}-T_{b}\right) \alpha E\left(b^{2}\left(r^{2}-a^{2}\right) \ln b+a^{2} \ln a(b-r)(b+r)+r^{2}(a-b)(a+b) \ln r\right)}{2(v-1) r^{2}(a-b)(a+b)(\ln a-\ln b)} \\
\sigma_{\theta}(r)= & \frac{\left(T_{a}-T_{b}\right) \alpha E\left(a^{2} \ln a\left(-\left(b^{2}+r^{2}\right)\right)+b^{2}\left(a^{2}+r^{2}\right) \ln b+r^{2}(a-b)(a+b)(\ln r+1)\right)}{2(v-1) r^{2}(a-b)(a+b)(\ln a-\ln b)}
\end{aligned}
$$

In equations (28) stress formulas coincides with the literature [7]. However an error is found in the definitions of those stresses in Reference [8]. Solutions in Reference [8] is unfortunately employed in Reference [9]. The analytical formulas, again derived in the present study, for the radial displacements and stresses due to mechanical loads such as internal/external pressure and rotation at a constant angular velocity are presented below for the sake of the completeness of the study.

$$
\begin{gathered}
u_{r}(r)=\left\{-\frac{a^{2}(v+1) p_{a}\left(b^{2}-2 v r^{2}+r^{2}\right)}{r\left(a^{2}-b^{2}\right) E}\right\}+\left\{\frac{b^{2}(v+1) p_{b}\left(a^{2}-2 v r^{2}+r^{2}\right)}{r\left(a^{2}-b^{2}\right) E}\right\} \\
\sigma_{r}(r)=\left\{\frac{a^{2} p_{a}\left(b^{2}-r^{2}\right)}{r^{2}\left(a^{2}-b^{2}\right)}\right\}+\left\{\frac{b^{2}(a-r)(a+r) p_{b}}{r^{2}\left(b^{2}-a^{2}\right)}\right\} \\
\sigma_{\theta}(r)=\left\{-\frac{a^{2} p_{a}\left(b^{2}+r^{2}\right)}{r^{2}\left(a^{2}-b^{2}\right)}\right\}+\left\{\frac{b^{2}\left(a^{2}+r^{2}\right) p_{b}}{r^{2}\left(a^{2}-b^{2}\right)}\right\} \\
u_{r}(r)=\left\{\frac{(v+1) \omega^{2} \rho\left(a^{2}(2 v-3)\left(b^{2}+(1-2 v) r^{2}\right)-(2 v-1) r^{2}\left(b^{2}(2 v-3)+r^{2}\right)\right)}{8(v-1) r E}\right\} \\
\sigma_{\theta}(r)=\left\{\frac{\omega^{2} \rho\left(a^{2}(2 v-3)\left(b^{2}+r^{2}\right)+r^{2}\left(b^{2}(2 v-3)+(2 v+1) r^{2}\right)\right)}{8(v-1) r^{2}}\right\}
\end{gathered}
$$


Table 1. Material properties for cylinders

\begin{tabular}{l|lrrrrr}
\hline & \multicolumn{1}{|c}{ METALS } & $E(\mathrm{GPa})$ & $\rho\left(\mathrm{kg} / \mathrm{m}^{3}\right)$ & $v$ & $k(\mathrm{~W} / \mathrm{mK})$ & $\alpha(1 / \mathrm{K})$ \\
\hline Metals & Titanium (Ti-6Al-4V) & 122.557 & 2370 & 0.29 & 13.723 & $7.579 \times 10^{-6}$ \\
& Aluminum (Al) & 70 & 2700 & 0.3 & 204 & $23 \times 10^{-6}$ \\
& Nickel (Ni) & 199.5 & 8900 & 0.3 & 90.7 & $13.3 \times 10^{-6}$ \\
& Stainless-Steel (SUS304) & 201.04 & 7800 & 0.3262 & 15.379 & $12.33 \times 10^{-6}$ \\
\hline Ceramics & Silicon-Nitride $\left(\mathrm{Si}_{3} \mathrm{~N}_{4}\right)$ & 348.43 & 4429 & 0.24 & 1.209 & $5.8723 \times 10^{-6}$ \\
& Zirconium-Oxide $\left(\mathrm{ZrO}_{2}\right)$ & 116.4 & 3657 & 0.3 & 1.78 & $8.7 \times 10^{-6}$ \\
& Aluminum-Oxide $\left(\mathrm{Al}_{2} \mathrm{O}_{3}\right)$ & 393 & 3970 & 0.3 & 30.1 & $8.8 \times 10^{-6}$ \\
\hline
\end{tabular}

For numerical example, geometrical and material properties of the cylindrical vessel are assumed to be: $a=0.8 \mathrm{~m} ; b=1.0 \mathrm{~m}$. Variation of the displacements and stresses induced by thermal loads at different temperature differences is illustrated in Figs. 6-7 for both ceramics and metallic materials whose properties are given in Table 1. From these figures it is observed that the characteristics of the curves of the elastic quantities are similar for both ceramics and metals since they are both isotropic and homogeneous: The radial displacement gradually increases with increasing radial coordinate. The maximum thermo-elastic radial displacement is observed at the vicinity of the middle surface. The thermo-elastic radial stresses are compression in nature. The maximum hoop stresses are observed at the inner surface of the cylindrical vessel. The thermo-elastic hoop stresses are gradually changed their signs from inside surface to the outer surface. The numerical values of the hoop stresses are 10-times more than radial stresses. So the hoop stresses become leading in the thermo-elastic analysis.
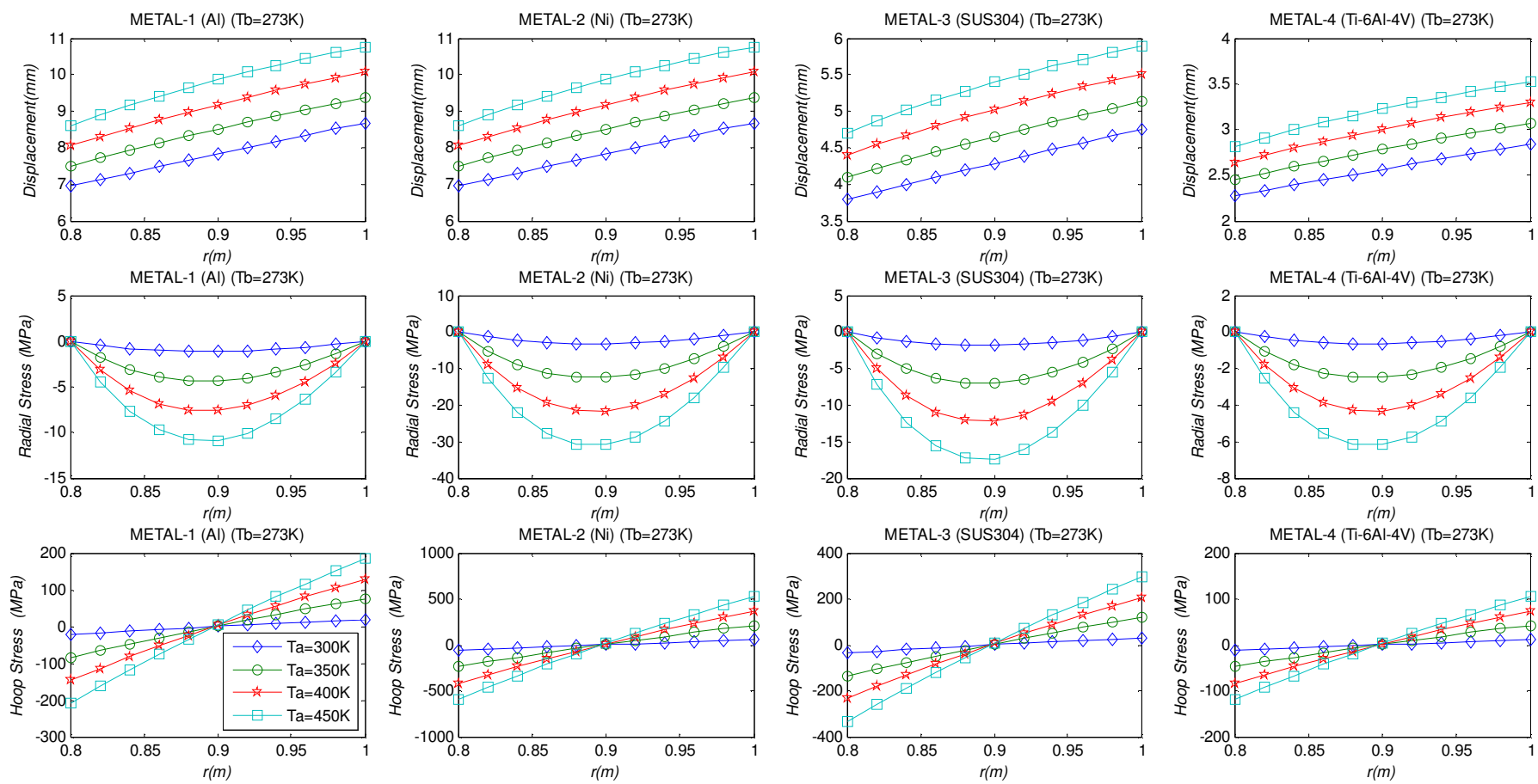

Fig. 6. Thermo-elastic radial displacement and the radial and hoop stresses for cylindrical vessels made of different metallic materials 

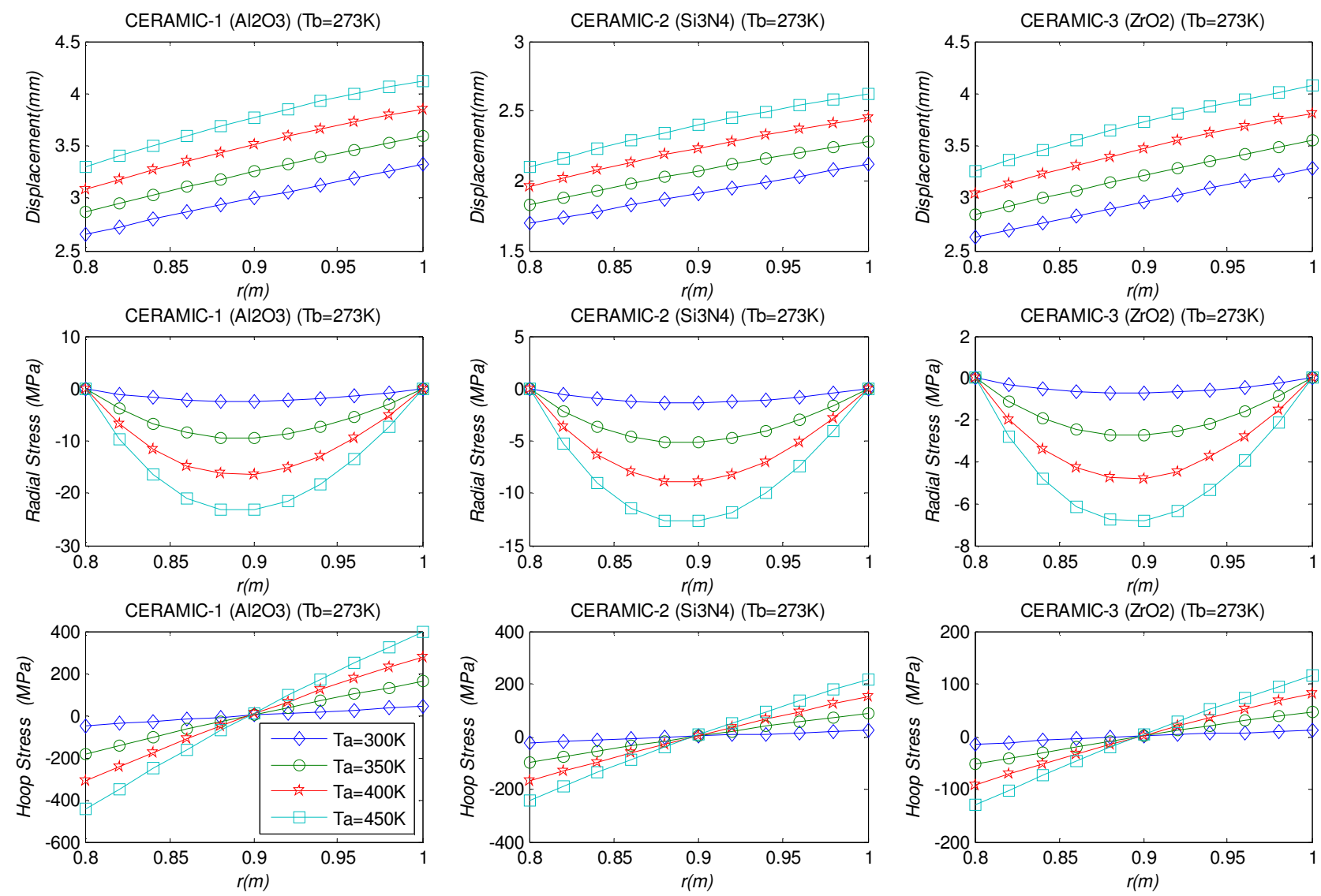

Fig. 7. Thermo-elastic radial displacement and the radial and hoop stresses for cylindrical vessels made of different ceramic materials

As expected, in a thermo-elastic analysis, the ceramic materials are more strength to the metallic materials. However, thermo-elastic behavior of a titanium-alloy is very similar to a zirconia. The titanium-alloy offers smaller displacements than the zirconia.

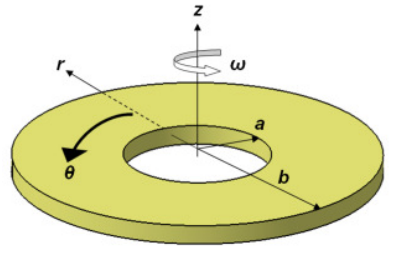

$\mathrm{BC}=1$ $\left\{\sigma_{r}(a)=0\right.$ and $\left.\sigma_{r}(b)=0\right\}$

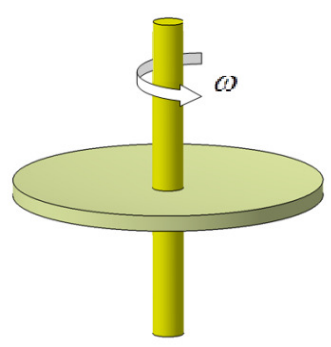

$\mathrm{BC}=2$ $\left\{u_{r}(a)=0\right.$ and $\left.\sigma_{r}(b)=0\right\}$

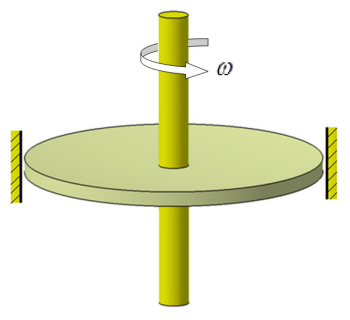

$\mathrm{BC}=3$

$\left\{u_{r}(a)=0\right.$ and $\left.u_{r}(b)=0\right\}$

Fig. 8. Boundary conditions considered for discs 


\section{Discs at Different Boundary Conditions}

In a polar coordinate system, $(r, \theta)$, axisymmetric field equations are as follows

$$
\begin{gathered}
\varepsilon_{r}(r)=u_{r}{ }^{\prime}(r) ; \quad \varepsilon_{\theta}(r)=\frac{u_{r}(r)}{r} ; \quad \gamma_{r \theta}(r)=0 \\
\sigma_{r}(r)=C_{11} \varepsilon_{r}(r)+C_{12} \varepsilon_{\theta}(r)-\left(C_{11}+C_{12}\right) \alpha \Delta T(r)=C_{11} \varepsilon_{r}(r)+\lambda C_{11} \varepsilon_{\theta}(r)-(1+\lambda) C_{11} \alpha \Delta T(r) \\
\sigma_{\theta}(r)=C_{12} \varepsilon_{r}(r)+C_{11} \varepsilon_{\theta}(r)-\left(C_{11}+C_{12}\right) \alpha \Delta T(r)=\lambda C_{11} \varepsilon_{r}(r)+C_{11} \varepsilon_{\theta}(r)-(1+\lambda) C_{11} \alpha \Delta T(r) \\
C_{11}=\frac{E}{(1-v)^{2}} ; \quad C_{12}=v C_{11}=\lambda C_{11}
\end{gathered}
$$

From the above field equations, the following Navier differential equation which governs the thermomechanical behavior of the uniform disc is obtained.

$$
u_{r}^{\prime \prime}(r)+\frac{1}{r} u_{r}^{\prime}(r)-\frac{1}{r^{2}} u_{r}(r)=-\frac{\rho \omega^{2} r}{C_{11}}+(1+\lambda) \alpha T^{\prime}(r)
$$

As stated above, temperature distribution for both discs and cylindrical vessels obey the same differential equations. So, from Eq. (26) the following is rewritten under the first kind boundary conditions

$$
T(r)_{C y l i n d e r \text { and Disc }}=\ln r \Theta_{1}+\Theta_{2}=\ln r \frac{T_{a}-T_{b}}{\ln a-\ln b}+\frac{-\ln b T_{a}+\ln a T_{b}}{\ln a-\ln b}
$$

In order to study thermo-elastic analysis alone of such structures, the rotation is omitted in Eq. (32).

$$
u_{r}^{\prime \prime}(r)+\frac{1}{r} u_{r}^{\prime}(r)-\frac{1}{r^{2}} u_{r}(r)=(1+v) \alpha \frac{\Theta_{1}}{r}=(1+v) \frac{\alpha}{r}\left(\frac{T_{a}-T_{b}}{\ln a-\ln b}\right)
$$

In the present work, the above differential equation is solved for each boundary condition given in Fig. 8 and the results are presented in Table 2. As ease of reference, the analytical formulas in Reference [10] for the uniform discs subjected to the mechanical loads are presented for different boundary conditions in the Appendix.

For a numerical study, geometrical and material properties of the disc are assumed to be: $a=0.1 \mathrm{~m}$; $b=1.0 \mathrm{~m}, \quad E=209.2 \mathrm{GPa} ; \quad v=0.29 ; \sigma_{\text {yield }}=700 \mathrm{MPa} ; \alpha=10.5810^{-6} 1 /^{\circ} \mathrm{C}$. Variation of the displacements and stresses induced by thermal loads is illustrated in Fig. 9 under different boundary conditions and for different temperature differences. From Fig. 9 it is observed that the radial displacement gradually increases with increasing $b / a$ ratios for $B C=1$ and $B C=2$. The maximum radial displacement is observed at the outer surface for both $B C=1$ and $B C=2$ while it is at the vicinity of the middle surface for $B C=3 . B C=1$ and $B C=3$ present radial stress as compression in nature while $B C=2$ offers radial stress in tension. The maximum radial stress is observed at the inner surface for $B C=2$, at the close to the inner surface for the others. From Fig. 9, for all types of boundary conditions, maximum hoop stress is observed at the inner surface of the disc. Hoop stresses are gradually changed their signs from inside surface to the outer surface. 

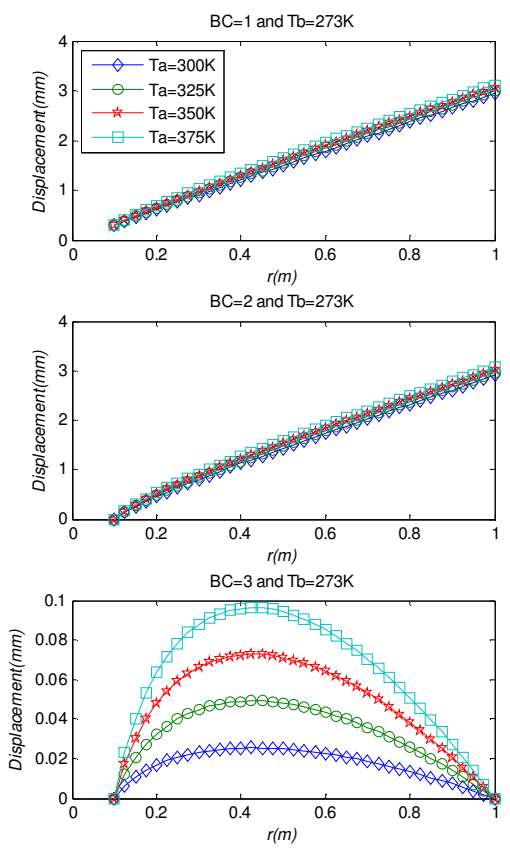
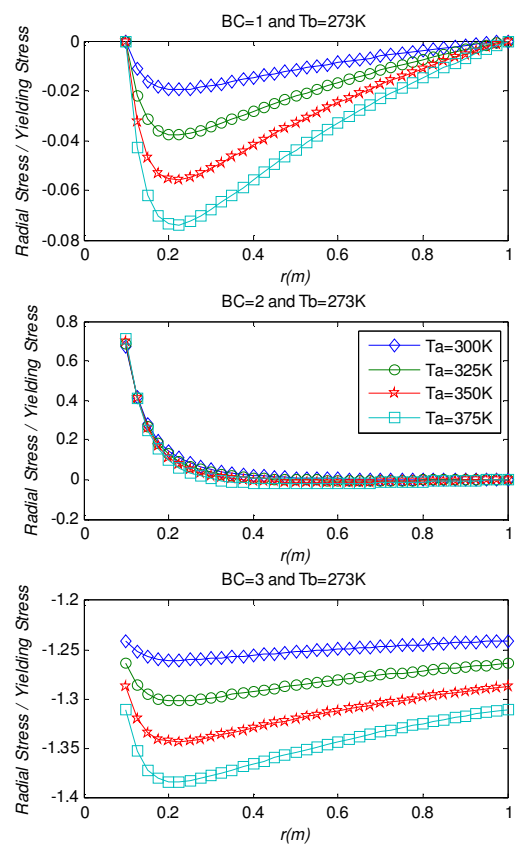
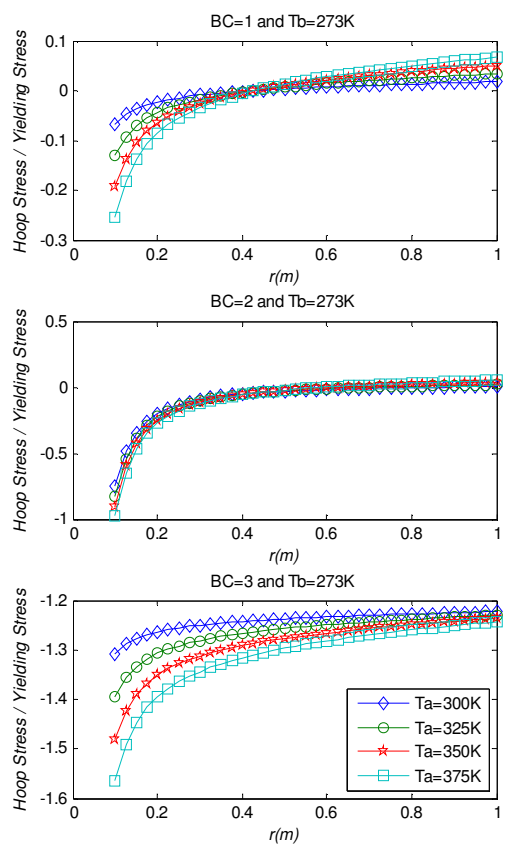

Fig. 9. Thermo-elastic behavior of a rotating disc at different boundary conditions

As stated above, some existing formulas in the literature contain some errors. Poworoznek [8] conducted an analytical study for cylindrical pressure vessels based on the theory proposed by Timoshenko [11]. He suggested some analytical formulas for both hollow cylinders (plain strain) and hollow discs (plain stress) for $B C=1$.

$$
\begin{gathered}
\left(\sigma_{r}\right)_{\text {Poworoznek } / \text { ISC }}=\frac{E \alpha T_{a}}{2(1-v) \ln \left(\frac{b}{a}\right)}\left(-\ln \left(\frac{b}{r}\right)-\frac{a^{2}}{b^{2}-a^{2}}\left(1-\frac{b^{2}}{r^{2}}\right) \ln \left(\frac{b}{a}\right)\right) \\
\left(\sigma_{\theta}\right)_{\text {Poworoznek/DISC }+ \text { CYLINDER }}=\frac{E \alpha T_{a}}{2 \ln \left(\frac{b}{a}\right)}\left(1-\ln \left(\frac{b}{r}\right)-\frac{a^{2}}{b^{2}-a^{2}}\left(1+\frac{b^{2}}{r^{2}}\right) \ln \left(\frac{b}{a}\right)\right) \\
\left(\sigma_{r}\right)_{\text {Poworoznek/CYLINDER }}=\frac{E \alpha T_{a}}{2 \ln \left(\frac{b}{a}\right)}\left(-\ln \left(\frac{b}{r}\right)-\frac{a^{2}}{b^{2}-a^{2}}\left(1-\frac{b^{2}}{r^{2}}\right) \ln \left(\frac{b}{a}\right)\right)
\end{gathered}
$$

Let's re-consider analytical formulas derived in this study for the radial and hoop stresses for discs (Table 2) and cylinders (Eq. (28)) under $B C=1$. Comparison shows that there are some syntax errors in those formulas suggested by Poworoznek [8] as follows

$$
\begin{gathered}
\left(\sigma_{r}\right)_{\text {Present } / D I S C}=(1-v)\left(\sigma_{r}\right)_{\text {Poworoznek } / D I S C} \\
\left(\sigma_{\theta}\right)_{\text {Present } / D I S C}=\left(\sigma_{\theta}\right)_{\text {Poworoznek } / D I S C} \\
\left(\sigma_{r}\right)_{\text {Present } / \text { CYLINDER }}=\frac{1}{(1-v)}\left(\sigma_{r}\right)_{\text {Poworoznek } / \text { CYLINDER }} \\
\left(\sigma_{\theta}\right)_{\text {Present } / C Y L I N D E R}=\frac{1}{(1-v)}\left(\sigma_{\theta}\right)_{\text {Poworoznek/CYLINDER }}
\end{gathered}
$$




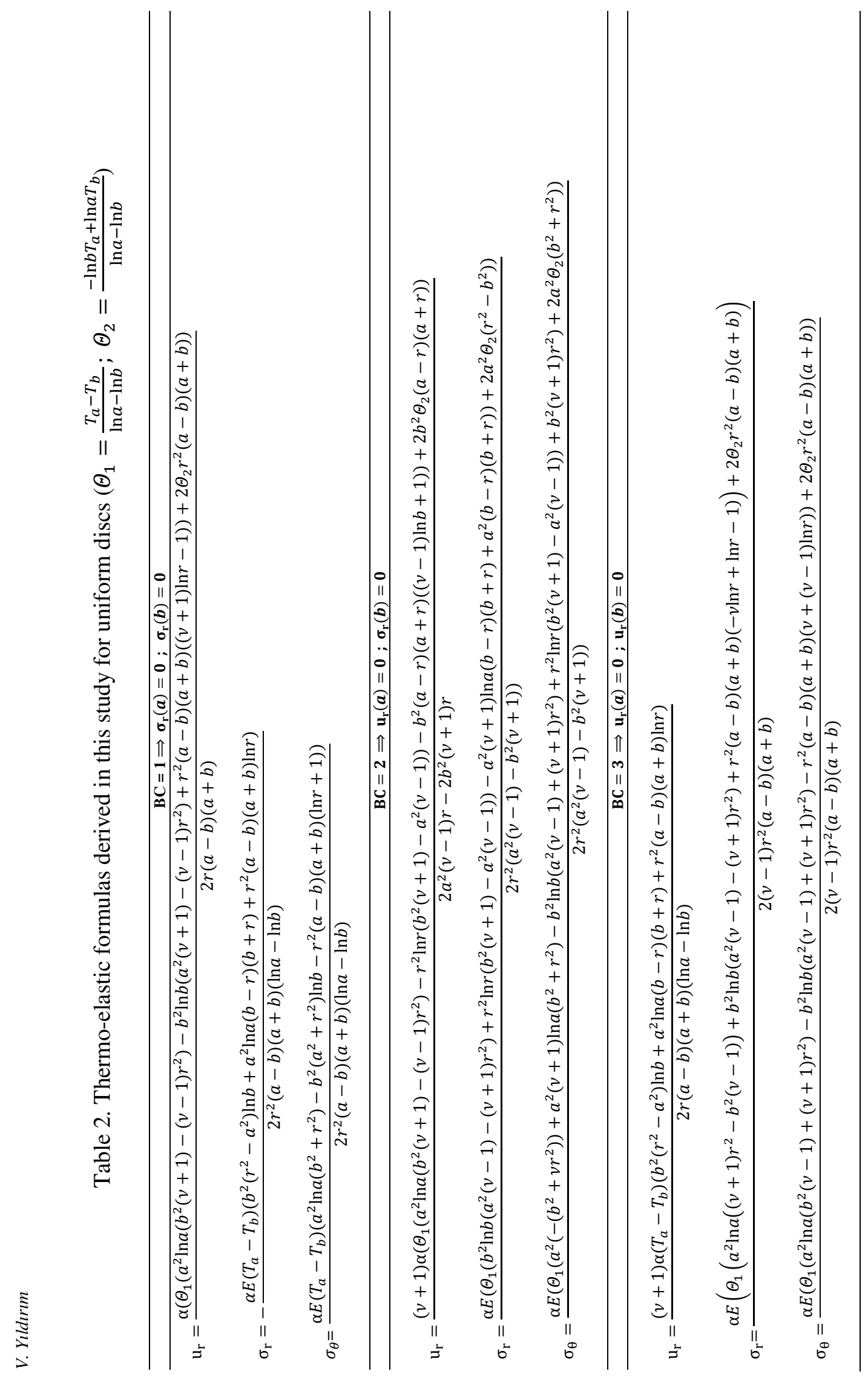


Before anything else, it is not proper to get the identical result for the hoop stresses in both plane strain and plane stress conditions as in Reference [8] while the radial stresses are found somewhat different for cylinders and discs. The author thinks that there must be some typing errors or some confusion between the elastic constants of plane stress and plain stress cases in those formulas in Reference [8].

To study the thermo-elastic behavior of the uniform discs under plane stress assumption the following differential equation should be used (See Eq. (32)).

$$
\begin{gathered}
u_{r}{ }^{\prime \prime}(r)+\frac{1}{r} u_{r}^{\prime}(r)-\frac{1}{r^{2}} u_{r}(r)=\left(1+\lambda_{\text {Plane-Stress }}\right) \alpha T^{\prime}(r) \\
\lambda_{\text {Plane-Stress }}=v
\end{gathered}
$$

Under plane strain assumption, the following differential equation governing the thermo-elastic behavior of the cylindrical structures should be used.

$$
\begin{gathered}
u_{r}^{\prime \prime}(r)+\frac{1}{r} u_{r}^{\prime}(r)-\frac{1}{r^{2}} u_{r}(r)=\left(1+2 \lambda_{\text {Plane-Strain }}\right) \alpha T^{\prime}(r) \\
\lambda_{\text {Plane-Strain }}=\frac{v}{1-v}
\end{gathered}
$$

Temperature distributions along the radial direction for both cylinders and uniform discs are identical.

$$
T(r)_{\text {cylinder and Disc }}=\ln r \Theta_{1}+\Theta_{2}
$$

From the above it is revealed that it is possible to confuse easily with the elasticity constants in the formulation. The present results for cylinders exactly coincides with the literature [7].

To gain insight into the issue in question, an additional numerical example is performed for both the discs and cylindrical vessels having the same inner and outer radii $(a=0.5 \mathrm{~m}, b=1 \mathrm{~m})$ for $B C=1$. The results are shown in Fig. 10 in a comparative manner by using the same axis-scales. From the overall picture the characteristics of the curves are similar to each other. However numerical values of the quantities are not the same. For example, the same temperature difference results in higher stresses in cylinders than discs.

Finally, it is possible to obtain plane-stress formulas from the plane strain formulas by using appropriate coefficients. The converse is also true. In the elementary elasticity theory those coefficients are given for mechanical loads such as rotation and internal/external pressures. For instance, if one replace formally $v$ with $\frac{v}{1-v}$, and $E$ with $\frac{E}{1-v^{2}}$ he may get the results for the plain-strain case from the plane stress solutions. As it is known $v$ should be replaced formally with $\frac{v}{1+v}$, and $E$ is to be replaced with $\frac{E(1+2 v)}{(1+v)^{2}}$ to get the plane stress results from the plain strain solutions. However this does not work alone for thermo-elastic analysis. 
DISC $(\mathrm{Tb}=300 \mathrm{~K})$

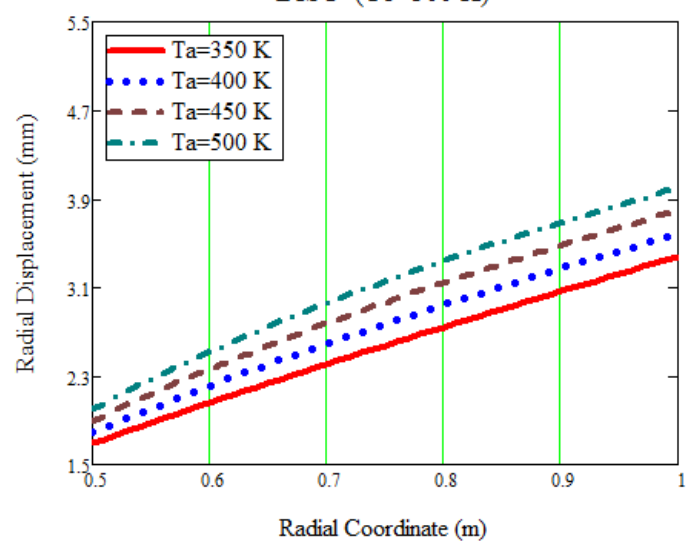

DISC $(\mathrm{Tb}=300 \mathrm{~K})$

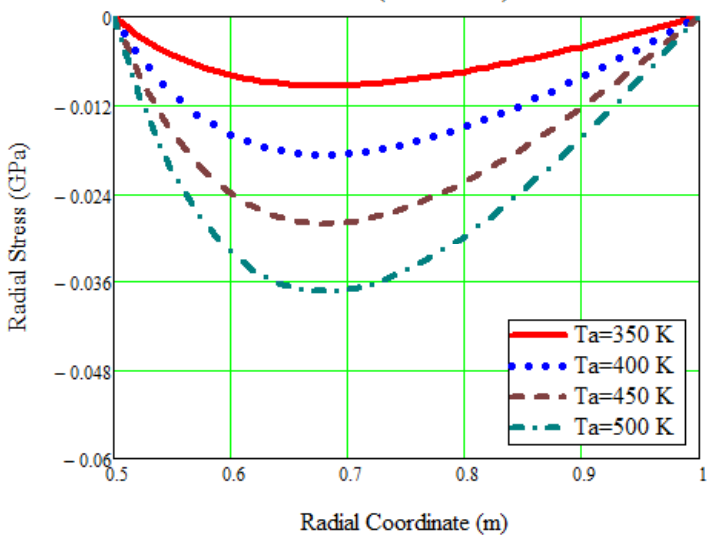

$\operatorname{DISC}(\mathrm{Tb}=300 \mathrm{~K})$

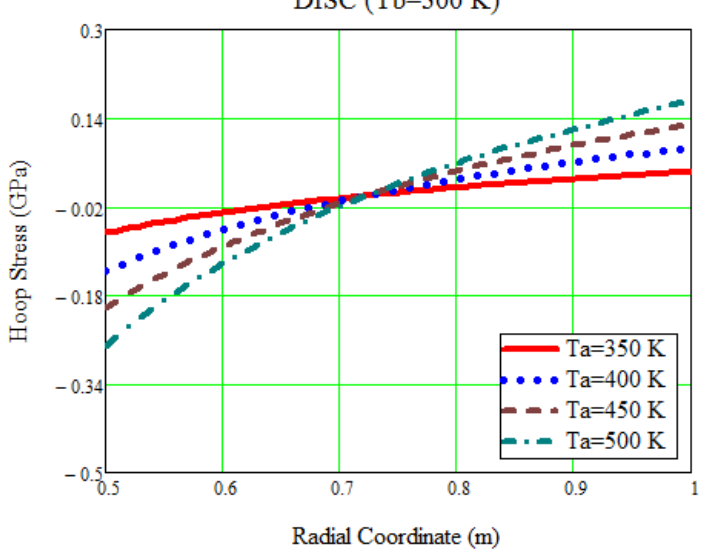

CYLINDER $(\mathrm{Tb}=300 \mathrm{~K})$

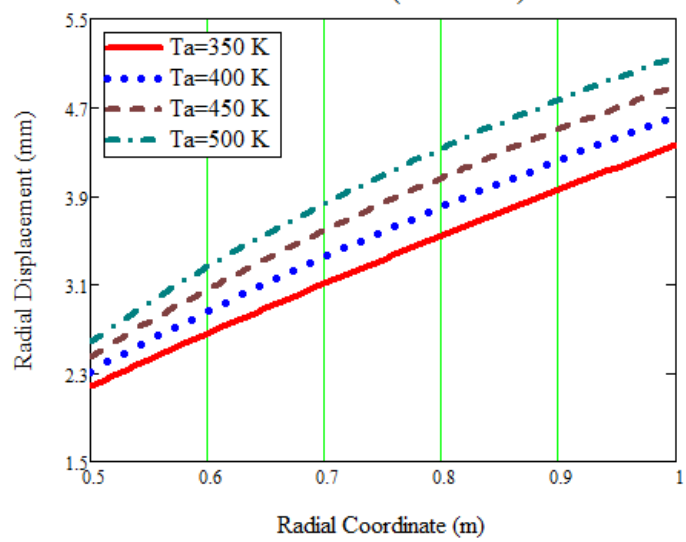

CYLINDER $(\mathrm{Tb}=300 \mathrm{~K})$

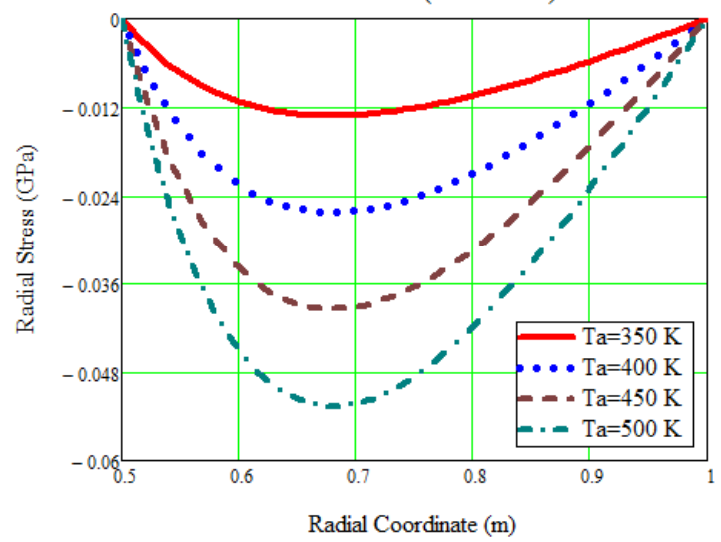

CYLINDER $(\mathrm{Tb}=300 \mathrm{~K})$

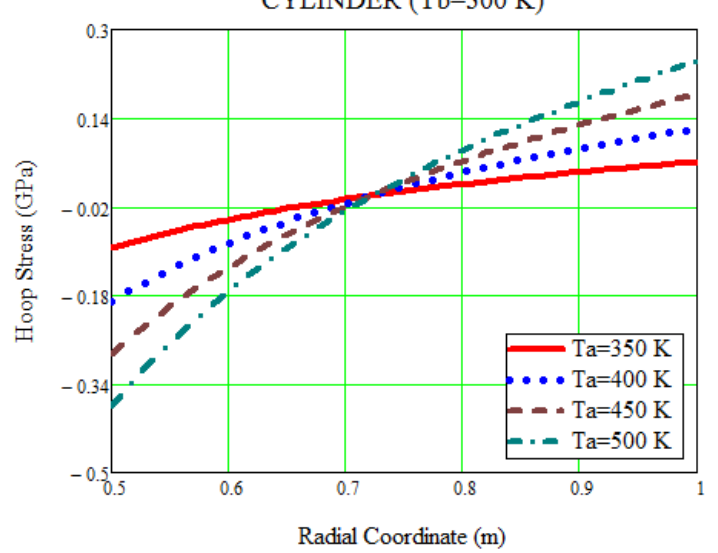

Fig. 10. Comparison of results for discs and cylinders $(a=0.5 m, b=1 m)$ under $\mathrm{BC}=1$ 


\section{Conclusions}

In this study thermo-mechanical analysis of annular structures made of a homogeneous and isotropic linear elastic material is handled analytically under different boundary conditions. The closed form formulas for the radial stress, hoop stress and the radial displacement are derived for each boundary condition and for each structural type. Apart from those, some muddles in the formulation of both cylinders and discs are clarified.

For the spherical rotating vessel with $p_{a}=200 \mathrm{MPa}, \omega=100 \frac{\mathrm{rad}}{\mathrm{s}}, T_{a}=300 \mathrm{~K}, \mathrm{~T}_{b}=273 \mathrm{~K}$, it is observed from Figs. 3-5 that

- Maximum radial displacement occurs at the inner surface for both pressure and centrifugal loads while it is located at the outer surface for thermal loads. For the given problem, thermal radial displacement are much excessive than mechanical load induced radial displacements.

- If radial stresses are considered, its maximum value is at the inner surface as in compression under pressure loading, at the mid-surface for both centrifugal force and thermal loads.

- As to the hoop stress, it reaches its maximum value at the inner surface as in tension for mechanical loads and it is also maximum at the inner surface as in compression for thermal loads. This contributes the almost uniform distribution of the total hoop stress along the thickness.

- The equivalent maximum stress is located at the inner surface due to all loadings, namely pressure, centrifugal force and thermal loads.

For the cylinders it is observed from Fig. 6 that the radial displacement progressively increases with increasing radial coordinate. The maximum thermo-elastic compressional radial displacement is examined at the vicinity of the middle surface. The maximum hoop stresses are watched at the inner surface of the cylindrical vessel. The thermo-elastic hoop stresses are in compression at the inner surface while they are in tension at the outer surface. The numerical values of the hoop stresses are nearly 10-times more than radial stresses. So the hoop stresses are guiding stresses in the thermo-elastic analysis.

The thermo-elastic behavior of stress-free discs is very similar to cylindrical vessels. However the same inner and outer radius together with the same temperature difference yield higher stresses in cylinders than stress-free discs. For other types of discs attached a shaft at its center (for $\mathrm{BC}=2$ and $\mathrm{BC}=3$ ) have much higher hoop stresses at the inner surface as in compression due to thermal loads.

By using the closed-form formulas offered in the present study, such structures may be tailored to the user's need. The author also hopes that this study may form an infallible all-in-one source for the readers studying the linear elastic behavior of such structures made of an isotropic and homogeneous material under thermal and mechanical loads. 
APPENDIX: Displacement and stresses of uniform isotropic and homogeneous discs subjected to mechanical loads [10] ( $p_{a}=$ Inner pressure, $p_{b}=$ Outer pressure)

$$
\begin{aligned}
& \begin{array}{l}
\sigma_{r}(a)=-p_{a} \\
\sigma_{r}(b)=-p_{b}
\end{array} \quad u_{r}=-\frac{a^{2} p_{a}\left(b^{2}(v+1)-(v-1) r^{2}\right)}{\operatorname{Er}\left(a^{2}-b^{2}\right)}+\frac{b^{2} p_{b}\left(a^{2}(v+1)-(v-1) r^{2}\right)}{\operatorname{Er}\left(a^{2}-b^{2}\right)} \\
& \sigma_{r}=\frac{a^{2} p_{a}\left(b^{2}-r^{2}\right)}{r^{2}\left(a^{2}-b^{2}\right)}+\frac{b^{2} p_{b}(a-r)(a+r)}{r^{2}\left(b^{2}-a^{2}\right)} \\
& \sigma_{\theta}=-\frac{a^{2} p_{a}\left(b^{2}+r^{2}\right)}{r^{2}\left(a^{2}-b^{2}\right)}+\frac{b^{2} p_{b}\left(a^{2}+r^{2}\right)}{r^{2}\left(a^{2}-b^{2}\right)} \\
& \sigma_{r}(a)=0 \\
& \sigma_{r}(b)=0 \\
& u_{r}=\frac{\rho \omega^{2}\left(a^{2}(v+3)\left(b^{2}(v+1)-(v-1) r^{2}\right)-(v-1) r^{2}\left(b^{2}(v+3)-(v+1) r^{2}\right)\right)}{8 E r} \\
& \sigma_{r}=\frac{\rho \omega^{2}(v+3)\left(a^{2}-r^{2}\right)\left(r^{2}-b^{2}\right)}{8 r^{2}} \\
& \sigma_{\theta}=\frac{\rho \omega^{2}\left(a^{2}(v+3)\left(b^{2}+r^{2}\right)+r^{2}\left(b^{2}(v+3)-(3 v+1) r^{2}\right)\right)}{8 r^{2}} \\
& u_{r}(a)=0 \quad u_{r}=\frac{\omega^{2} \rho\left(a^{2}(v+3)\left(b^{2}(v+1)-(v-1) r^{2}\right)-(v-1) r^{2}\left(b^{2}(v+3)-(v+1) r^{2}\right)\right)}{8 r E} \\
& \sigma_{r}(b)=0 \\
& \sigma_{r}=\frac{(v+3) \omega^{2}(a-r)(a+r)\left(r^{2}-b^{2}\right) \rho}{8 r^{2}} \\
& \sigma_{\theta}=\frac{\omega^{2} \rho\left(a^{2}(v+3)\left(b^{2}+r^{2}\right)+r^{2}\left(b^{2}(v+3)-(3 v+1) r^{2}\right)\right)}{8 r^{2}} \\
& u_{r}(a)=0 \\
& u_{r}(b)=0 \\
& u_{r}=\frac{\left(v^{2}-1\right) \omega^{2}\left(r^{2}-a^{2}\right)\left(r^{2}-b^{2}\right) \rho}{8 r E} \\
& \sigma_{r}=\frac{\omega^{2} \rho\left(a^{2}\left((v+1) r^{2}-b^{2}(v-1)\right)+r^{2}\left(b^{2}(v+1)-(v+3) r^{2}\right)\right)}{8 r^{2}} \\
& \sigma_{\theta}=\frac{\omega^{2} \rho\left(a^{2}\left(b^{2}(v-1)+(v+1) r^{2}\right)+r^{2}\left(b^{2}(v+1)-(3 v+1) r^{2}\right)\right)}{8 r^{2}}
\end{aligned}
$$




$\begin{array}{ll}\begin{array}{c}\text { Notations } \\ a, b\end{array} & \text { Inner radius and outer radius, respectively } \\ C_{1}, C_{2} & \text { Integration constants } \\ C_{i j} & \text { elastic constants in Hooke's law } \\ E & \text { Young's modulus } \\ p_{a}, p_{b} & \text { Pressures at inner and outer surfaces, respectively } \\ r & \text { radial coordinate } \\ T_{a}, T_{b} & \text { temperature at the inner and outer surfaces, respectively } \\ u_{r} & \text { radial displacement } \\ \varepsilon_{r} & \text { radial strain } \\ \varepsilon_{\theta} & \text { tangential strain } \\ \alpha & \text { thermal expansion coefficient } \\ \gamma_{r \theta}, \gamma_{r \phi}, \gamma_{\theta \phi} & \text { engineering shear strain components } \\ \varnothing & \text { Azimuthal coordinate } \\ v & \text { Poisson's ratio } \\ \rho & \text { density of the vessel material } \\ \sigma_{r} & \text { radial stress } \\ \sigma_{\theta} & \text { hoop stress } \\ \theta & \text { tangential coordinate } \\ \omega & \text { constant angular velocity }(\mathrm{rad} / \mathrm{s})\end{array}$

\section{References}

[1] Bower, A. F., Applied Mechanics of Solids; Taylor and Francis, 2012.

[2] Young, W.C., Budynas, R.G., Roark's Formulas for Stress and Strain; McGrawHill, Seventh Edition, New York. 2002.

[3] Hetnarski, B., Eslami, M.R., Thermal Stresses-Advanced Theory and Applications; Springer, 2009.

[4] Nayak, P., Mondal, S.C., Nandi, A., Stress, strain and displacement of a functionally graded thick spherical vessel. International Journal of Engineering Science and Technology (IJEST), 3/4, 2659-2671, 2011.

[5] Chakrabarty, J., Theory of Plasticity; McGraw Hill, New York, 1998.

[6] Noda, N., Hetnarski, R.B., Tanigawa , Y., Thermal Stresses; Taylor and Francis, New York, 2003.

[7] Heat Transfer Problems and Thermal Stresses, FEM II. Comp. Lab, www.meil.pw.edu.pl/sms/content/download/24488/.../FEMII_LAB_THERM_v2.pdf

[8] Poworoznek, P.P., Elastic-Plastic Behavior of a Cylinder Subject to Mechanical and Thermal Loads; Rensselaer Polytechnic Institute, Master Thesis, Hartford, CT. 2008.

[9] Kanlıkama, B., Abuşoğlu, A., Güzelbey, İ.H. Coupled thermoelastic analysis of thick-walled pressurized cylinders. International Journal of Energy and Power Engineering, 2/2, 60-68. 2013.

[10] Yıldırım, V., Analytic solutions to power-law graded hyperbolic rotating discs subjected to different boundary conditions. International Journal of Engineering \& Applied Sciences (IJEAS), 8/1, 38-52, 2016.

[11] Timoshenko, S., Strength of Material Part II, Advanced Theory and Problems, 3rd Edition, D. Van Nostrand Company Inc., Princeton, NJ, 1956. 